\title{
Long Non-coding RNA HOTAIR Expression in Diffuse Large B-Cell Lymphoma: In Relation to Polycomb Repressive Complex Pathway Proteins and H3K27 Trimethylation
}

\begin{abstract}
Eun Ji Oh ${ }^{1,2 *} \cdot$ Soo Hee $\mathrm{Kim}^{1,3,4 *}$ Woo Ick Yang ${ }^{1}$. Young Hyeh $\mathrm{Ko}^{3}$ Sun Och Yoon ${ }^{1}$

'Department of Pathology, Yonsei University College of Medicine, Seoul; ' ${ }^{2}$ epartment of Pathology, Seoul St. Mary's Hospital, College of Medicine, The Catholic University of Korea, Seoul; '3epartment of Pathology, Samsung Medical Center, Sungkyunkwan University School of Medicine, Seoul; ${ }^{4}$ Anatomic Pathology Reference Lab, Seegene Medical Foundation, Seoul, Korea
\end{abstract}

Received: May 30, 2016

Accepted: June 6, 2016

Corresponding Author

Sun Och Yoon, MD, PhD

Department of Pathology, Yonsei University College

of Medicine, 50-1 Yonsei-ro, Seodaemun-gu, Seoul

03722, Korea

Tel: +82-2-2228-1763

Fax: +82-2-362-0860

E-mail: soyoon@yuhs.ac; revita@naver.com

*Eun Ji Oh and Soo Hee Kim contributed equally to this work.
Background: A long non-coding RNA hox transcript antisense intergenic RNA (HOTAIR) is involved in epigenetic regulation through chromatin remodeling by recruiting polycomb repressive complex 2 (PRC2) proteins (EZH2, SUZ12, and EED) that induce histone H3 trimethylation at lysine 27 (H3K27me3). Deregulation of c-MYC and interaction between c-MYC and EZH2 are well known in lymphomagenesis; however, little is known about the expression status of HOTAIR in diffuse large B-cell lymphomas (DLBCLs). Methods: The expression status of PRC2 (EZH2, SUZ12, and EED), H3K27me3, c-MYC, and BCL2 was analyzed using immunohistochemistry (n $=231$ ), and HOTAIR was investigated by a quantification real-time polymerase chain reaction method ( $n=164$ ) in DLBCLs. Results: The present study confirmed the positive correlation among PRC2 proteins, H3K27me3, and c-MYC in DLBCLs. Expression level of HOTAIR was also positively correlated to EZH2 ( $\mathrm{p}<.05$, respectively). Between c-MYC and HOTAIR, and between cMYC/BCL2 co-expression and HOTAIR, however, negative correlation was observed in DLBCLs $(\mathrm{p}<.05$, respectively). High level of $\mathrm{H} 3 \mathrm{~K} 27 \mathrm{me} 3$ was determined as an independent prognostic marker in poor overall survival (hazard ratio, 2.0; $p=.023$ ) of DLBCL patients. High expression of HOTAIR, however, was associated with favorable overall survival $(p=.004)$ in the univariate analysis, but the impact was not significant in the multivariate analysis. The favorable outcome of DLBCL with HOTAIR high expression levels may be related to the negative correlation with cMYC expression or c-MYC/BCL2 co-expression. Conclusions: HOTAIR expression could be one of possible mechanisms for inducing $\mathrm{H} 3 \mathrm{~K} 27$ me3 via EZH2-related $\mathrm{PRC} 2$ activation, and induced H3K27me3 may be strongly related to aggressive DLBCLs which show poor patient outcome.

Key Words: Lymphoma, large B-cell, diffuse; HOTAIR; hox transcript antisense intergenic RNA; Polycomb repressive complex2; Histone H3trimethylation at lysine 27
Long non-coding RNAs (lncRNAs) are molecules longer than 200 nucleotides that are not translated into proteins. The role of IncRNAs is mostly unknown. Recently, several types of cancer-related IncRNAs have been identified and studied in the field of translational research. ${ }^{1-4}$ According to recent studies, some IncRNAs are involved in the epigenetic regulation of protein coding genes. Hox transcript antisense intergenic RNA (HOTAIR) is one of most actively studied lncRNAs. Overexpression of HOTAIR is known to occur in various solid tumors of esophagus, stomach, colon, liver, pancreas, lung, and breast and is related to poor prognosis in those tumors. ${ }^{3,5}$ HOTAIR is located within the bomeobox C (HOXC) gene cluster on chromosome12 and is co-expressed with the HOXC genes. HOTAIR is involved in chromatin remodeling through recruiting polycomb repressive complex 2 (PRC2; enhancer of zeste homolog 2 [EZH2], suppressor of zeste 12 homolog [SUZ12], and embryonic ectoderm development [EED]) and then inducing histone modification such as histone $\mathrm{H} 3$ trimethylation at lysine $27(\mathrm{H} 3 \mathrm{~K} 27 \mathrm{me} 3)$ at the promoter site of protein-coding genes. ${ }^{1-4}$ Chromatin remodeling and gene regulation via histone modification of the polycomb repressive complex is known to function in the development of embryonic stem cells as well as the development of many types of cancers including hematologic malignancies. ${ }^{6,7}$

In hematologic malignancies, especially in diffuse large $\mathrm{B}$ cell lymphomas (DLBCLs) and follicular lymphomas that are the most predominant lymphoma subtype, the deregulation of 
EZH2 methyltransferase is well known. In our previous study, the high level of global H3K27me3 in DLBCL was associated with poor patient prognosis. ${ }^{8}$ From these findings, it was suggested that HOTAIR might be involved in the PRC2-associated induction of $\mathrm{H} 3 \mathrm{~K} 27 \mathrm{me} 3$ in DLBCLs; however, an association with HOTAIR and DLBCL has not yet been described. In the present study, the expression status of HOTAIR was investigated in DLBCL, and the association with PRC2 and H3K27me3 was analyzed.

\section{MATERIALS AND METHODS}

\section{Patients and clinical data}

A total of 231 cases of DLBCL treated with R-CHOP (rituximab, cyclophosphamide, doxorubicin, vincristine, and prednisone) or R-CHOP-like (with or without radiotherapy or surgery) chemotherapy were selected for the study. Cases were retrieved from the archival files from the Department of Pathology, Severance Hospital, from 2005 to 2011. All cases were independently reviewed by two pathologists (S.O.Y. and S.H.K.) based on current World Health Organization criteria, ${ }^{9}$ and discordant cases were consulted to other expert hematopathologists. In HOTAIR expression analysis, 164 cases were selected from the above 231 cases and investigated after quality assessment of extracted RNA. Clinical data were obtained from medical records. All study protocols were performed according to the ethical guidelines of the "World Medical Association Declaration of Helsinki-Ethical Principles for Medical Research Involving Human Subjects.” This study was approved by the Institutional Review Board of Severance Hospital.

\section{Analysis for HOTAIR expression}

Formalin fixed paraffin embedded (FFPE) tissue sections were prepared and stained with hematoxylin and eosin, and then the tumor areas were confirmed and marked under the microscope. The marked areas mainly contained packed tumor cells, and the stromal component was less than $10 \%$ of the marked area. The unstained slides of FFPE tissues were prepared after dissecting FFPE tissue blocks at 10-mm thickness using a microtome, and the marked area was scraped using a scalpel blade. Generally, three slices of tissue section per case were used for RNA extraction. Total RNA was isolated using an RNeasy FFPE Kit (Qiagen, Hilden, Germany) according to the supplier's instructions. Extracts of RNA were verified by measuring the ratios ofA260/ A280 and A260/A230 with a ND-1000 NanoDrop spectrophotometer (NanoDrop, Wilmington, DE, USA). Reverse tran- scription was performed using a QuantiTect Reverse Transcription kit (Qiagen). The expression patterns of HOTAIR were assayed by relative quantification using expression of the housekeeping gene glyceraldehyde-3-phosphate dehydrogenase $(G A P D H)$. Primers for HOTAIR and GAPDH were as follows: HOTAIR (forward, 5'-AGCCAGAGGAGGGAAGAGAG-3'; reverse, 5'-TCCCGTTCCCTAGATTTTCC-3') and GAPDH (forward, 5'-CAAATTCCATGGCACCGTCA-3'; reverse, 5'-ATCGCCCCACTTGATTTTGG-3'). Primers of HOTAIR were designed to detect all three transcript variants (transcript variant 1,3 , and 2). In brief, a $20 \mu \mathrm{L}$ mixture containing $1.0 \mu \mathrm{L}$ of cDNA, power SYBR Green PCR Master Mix (Applied Biosystems, Carlsbad, CA, USA), $1.0 \mu \mathrm{L}$ of $10 \mathrm{pmol} / \mu \mathrm{L}$ forward primer, $1.0 \mu \mathrm{L}$ of $10 \mathrm{pmol} / \mu \mathrm{L}$ reverse primer, and $7.0 \mu \mathrm{L}$ of tertiary distilled water was prepared. Amplification was performed using a Step One Plus Real-Time PCR instrument (Applied Biosystems) under the following conditions: denaturation at $94^{\circ} \mathrm{C}$ for 10 minutes, followed by 35 cycles of $94^{\circ} \mathrm{C}$ for 15 seconds, $55^{\circ} \mathrm{C}$ for 30 seconds, and $72^{\circ} \mathrm{C}$ for 30 seconds (fluorescence signal acquisition was performed at this phase). Immediately after amplification, melting curve analysis was performed for amplicon verification. All samples were analyzed in triplicate to confirm reproducibility. Using the Step One Plus Real-Time PCR System software ver. 2.1 (Applied Biosystems), the threshold cycle $(\mathrm{Ct}$, beginning of the polymerase chain reaction exponential phase) value of amplified HOTAIR was normalized versus that of amplified GAPDH $\left(2^{-d C}\right)$. After quality assessment of extracted RNA and expression analysis of the housekeeping gene GAPDH, 164 cases were finally selected for the analysis of HOTAIR expression. Normal palatine tonsil tissue was obtained from 10 cancer-free individuals and used as age-matched cancerfree controls. The cutoff value for high expression of HOTAIR $\left(H_{O T A I R^{\text {high }}}\right)$ was determined at the uppermost value among those of normal control tonsil tissues.

Tissue microarray preparation, immunohistochemistry, and analysis

The hematoxylin and eosin slides were reviewed and two representative core tissues of the tumor area were selected in each case. Core tissues ( $3 \mathrm{~mm}$ in diameter) were taken from the individual donor blocks and arranged in new recipient tissue microarray paraffin blocks using a trephine apparatus. Immunohistochemical staining and in situ hybridization were performed on 4- $\mu \mathrm{m}$ tissue microarray tissue sections. Immunohistochemistry of EZH2 (1:100, Invitrogen, Carlsbad, CA, USA), SUZ12 (1:50, Abcam, Cambridge, UK), EED (1:1,000, Abcam), H3K27me3 
(1:100, clone C36B11, Cell Signaling Technology, Beverly, MA, USA) was performed using the Ventana BenchMark XT Autostainer (Ventana Medical Systems, Tucson, AZ, USA). Immunohistochemistry for c-MYC (1:50, clone Y69, Abcam), BCL2 (1:50, Novocastra, Newcastle, UK), CD10 (1:100, Novocastra), BCL6 (RTU, Novocastra), and MUM1 (1:200, Cell Marque, Rocklin, CA, USA) was performed using the LEICA BONDIII Autostainer (Leica Biosystems, Newcastle Upon Tyne, UK). For in situ hybridization for Epstein-Barr virus (EBV), the INFORM EBER probe (Ventana Medical Systems) was used with the Ventana BenchMark XT Autostainer (Ventana Medical Systems) and ISH iVIEW Blue Detection kit (Ventana Medical Systems).

For EZH2, SUZ12, EED, and H3K27me3, staining intensity $(0$, no staining to weak; 1 , moderate to strong) and proportion of positive tumor cell nuclei $(0,<10 \% ; 1, \geq 10 \%$ and $<75 \% ; 2$, $\geq 75 \%$ ) were semiquantitatively graded as in the previous study. ${ }^{8}$ Based on the intensity multiplied by proportion, the protein ex- pression was scored as low (0), intermediate (1), or high level (2). For CD10, BCL6, and MUM1, the positive cutoff value was determined according to the Hans classification criteria ${ }^{10}$ and was considered positive if $\geq 30 \%$ of the tumor cells showed nuclear immunoreactivity for BCL6 and MUM1, and if $\geq 30 \%$ of cells showed membranous reactivity for $\mathrm{CD} 10$. Determination of the germinal center B-like (GCB) or non-GCB phenotype was based on the Hans algorithm 10.

For c-MYC and BCL2, staining intensity ( 0 , no staining to weak; 1 , moderate to strong) and areas of positive tumor cell nuclei by $10 \%$ increments ( 1 of $<10 \%$ to 10 of $90 \%-100 \%$ ) were semiquantitatively graded. The cutoff value for high expression was determined by log-rank tests (Mantel-Cox) for overall survival as in the previous report, and the threshold value was determined for c-MYC at a score $\geq 4$ ( $\geq 40 \%$ of tumor cells with moderate to strong expression), and BCL2 at a score $\geq 7$ ( $\geq 70 \%$ of tumor cells with moderate to strong expression). ${ }^{11,12}$ For EBV in situ hybridization, the threshold value was determined when
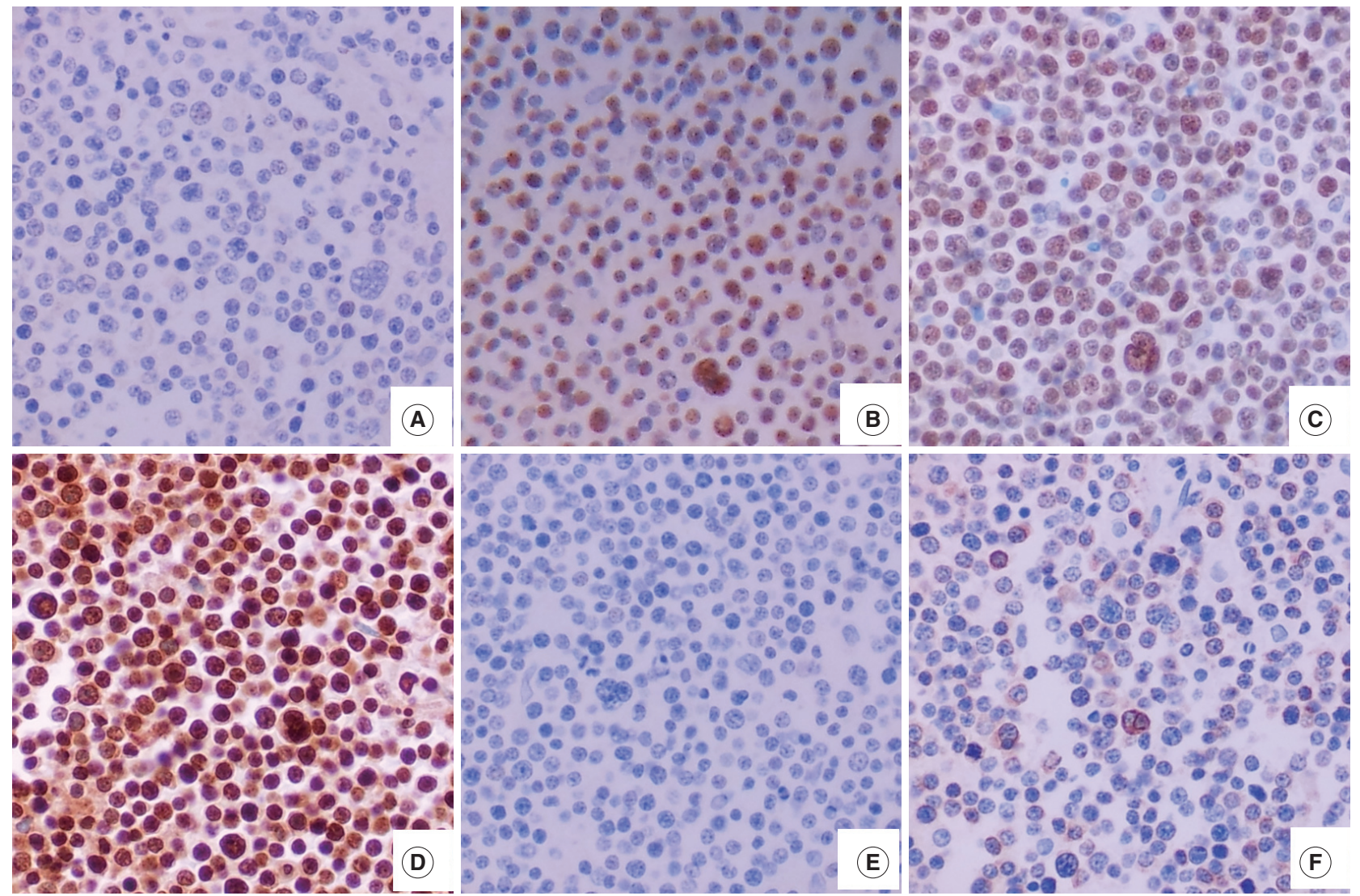

Fig. 1. A case of DLBCL with HOTAIR high (A-F) shows the various expression pattern of c-MYC (A), EZH2 (B), SUZ12 (C), EED (D), global H3K27me3 (E), and BCL2 (F). In a DLBCL case of HOTAIR high (A-F), many PRC2 protein (EZH2, SUZ12, and EED)-positive tumor cells show no expression of c-MYC and no mark of H3K27me3. BCL2 was negative in this case. DLBCL, diffuse large B-cell lymphoma; HOTAIR, hox transcript antisense intergenic RNA; EZH2, enhancer of zeste homolog 2; SUZ12, suppressor of zeste 12 homolog; EED, embryonic ectoderm development; PRC2, polycomb repressive complex 2. 
$\geq 10 \%$ of the tumor cells showed moderate to strong nuclear expression.

The features of expression of polycomb repressive complex proteins (EZH2, SUZ12, and EED), H3K27me3, c-MYC, and BLC2 in HOTAIR ${ }^{\text {high }}$ or HOTAIR ${ }^{\text {low }}$ cases are presented in Fig. 1.

\section{Statistical analysis}

The $t$ test and chi-square test were used to analyze the differences between the variables examined. Overall survival times were measured from the date of lymphoma diagnosis to the date of death or last follow-up visit. Patient survival rates were determined using the Kaplan-Meier method, and the differences in survival rates were compared using the log-rank test. Multivariate analysis was performed using the Cox proportional hazards
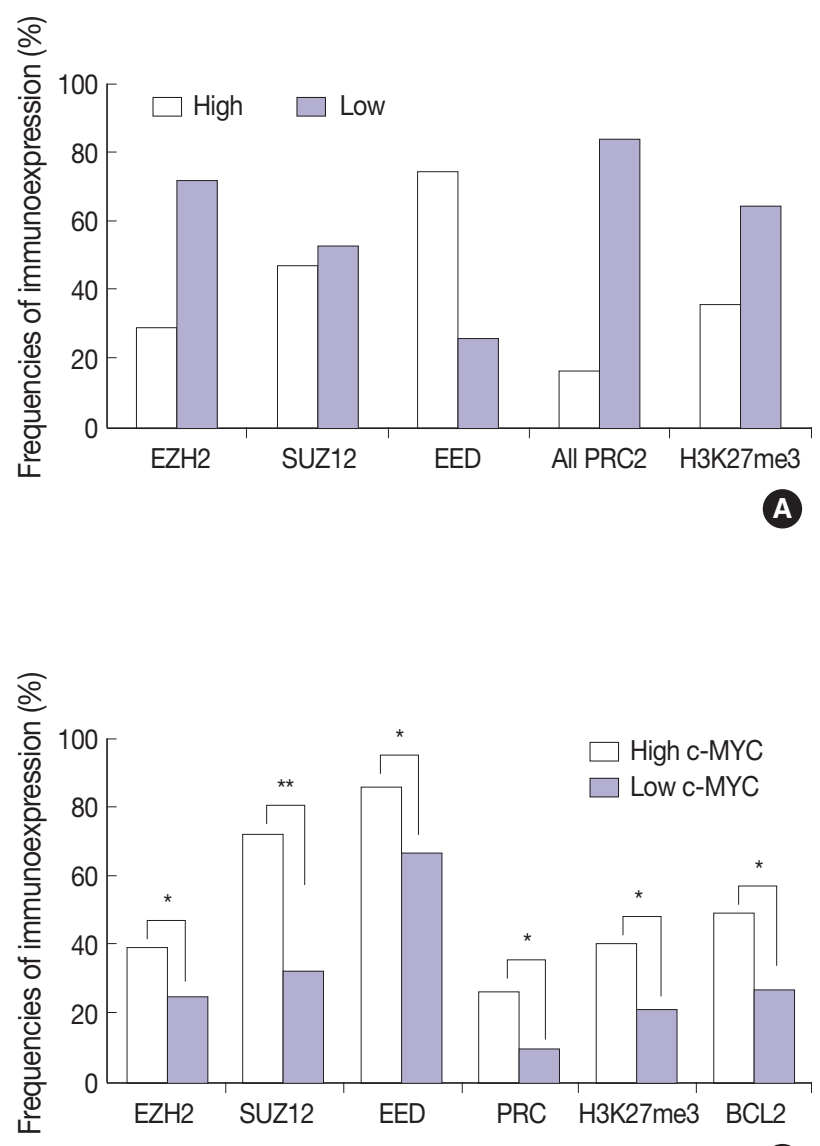

model. A two-sided p-value $<.05$ was considered to be statistically significant. When a two-sided p-value was $\leq .05$ and $<.10$, a trend toward statistical significance was considered. All statistical analyses were carried out using SPSS software ver. 20.0 for Windows (IBM Corp., Armonk, NY, USA).

\section{RESULTS}

The relations among PRC2 proteins, H3K27me3, c-MYC, BCL2, and HOTAIR

The DLBCL cases frequently showed high expression of PRC2 proteins and $\mathrm{H} 3 \mathrm{~K} 27 \mathrm{me} 3$ (28.9\%, 47.1\%, $74.3 \%$, and $35.7 \%$ for $\mathrm{EZH} 2^{\text {high }}$, SUZ12 ${ }^{\text {high }}$, EED ${ }^{\text {high }}$, and $\mathrm{H} 3 \mathrm{~K} 27 \mathrm{me}^{\text {high }}$, respectively). High expression of all PRC2 proteins (EZH2 $2^{\text {high/ }}$
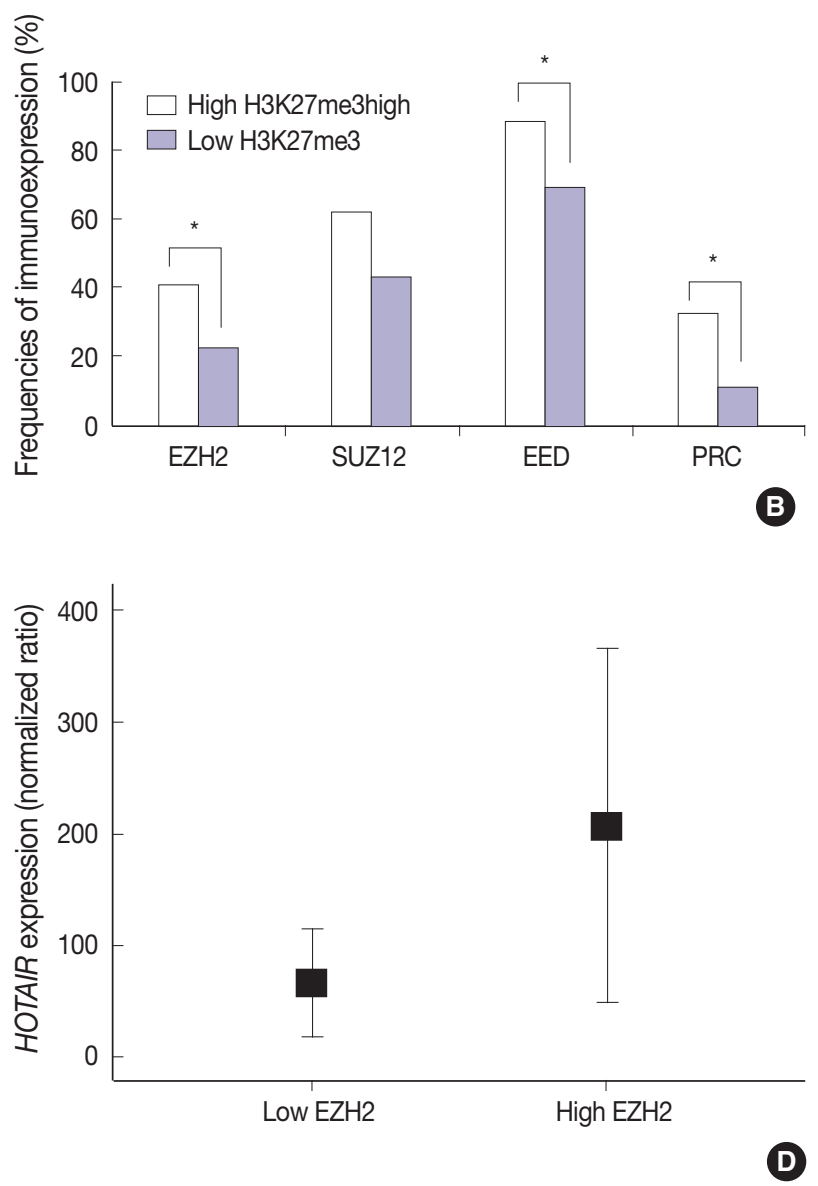

Fig. 2. (A) The DLBCL cases frequently show high expression of PRC2 proteins (EZH2, SUZ12, and EED), high expression of all PRC2 proteins (EZH2high/SUZ12 high/EED high), and high H3K27me3 level. (B) When compared to cases without high H3K27me3 expression (low H3K27me3), those with high H3K27me3 expression more frequently show high expression of EZH2, SUZ12, EED, and all PRC2 (EZH2 ${ }^{\text {high }} / \mathrm{SU}$ Z12 high/EED high). (C) When compared to cases without high c-MYC expression (low c-MYC), those with high c-MYC expression more frequently show high expression of EZH2, SUZ12, EED, all PRC2 (EZH2 high/SUZ12 high/EED high), H3K27me3, and BCL2. (D) The expression level of HOTAIR is significantly higher in cases with high EZH2 expression than those without high EZH2 expression (low EZH2). DLBCL, diffuse large B-cell lymphoma; HOTAIR, hox transcript antisense intergenic RNA; EZH2, enhancer of zeste homolog 2; SUZ12, suppressor of zeste 12 homolog; EED, embryonic ectoderm development; PRC2, polycomb repressive complex $2 .{ }^{*} p<.05,{ }^{* \star} p<.001$. 
SUZ12 ${ }^{\text {high }} / \mathrm{EED}^{\text {high }}$ ) was noted in $16.1 \%$ of cases (Fig. 2A). The expression of $\mathrm{H} 3 \mathrm{~K} 27 \mathrm{me} 3$ was positively correlated to PRC2 markers. When compared to cases of $\mathrm{H} 3 \mathrm{~K} 27 \mathrm{me} 3^{\text {low }}$, those of high $\mathrm{H} 3 \mathrm{~K} 27 \mathrm{me} 3$ expression more frequently showed high expression of EZH2 (41.2\% vs $22.2 \%, \mathrm{p}=.003)$, SUZ12 (62.5\% vs $43.0 \%, \mathrm{p}=.053), \operatorname{EED}(88.6 \%$ vs $69.7 \%, \mathrm{p}=.026)$, and all PRC2 (EZH2 $2^{\text {high }} / \mathrm{SUZ} 2^{\text {high }} / \mathrm{EED}^{\text {high }}$ ) $(32.3 \%$ vs $11.3 \%, \mathrm{p}=.005)$.

These results are summarized in Fig. 2B.

The expression of c-MYC was positively correlated to PRC2 markers and H3K27me3. When compared to cases of c-MY$\mathrm{C}^{\text {low }}$, those of high c-MYC expression more frequently showed high expression of EZH2 (39.1\% vs 24.6\%, p =.036), SUZ12 (72.2\% vs $32.1 \%, \mathrm{p}<.001), \operatorname{EED}(85.7 \%$ vs $66.7 \%, \mathrm{p}=.011)$, all PRC2 (EZH2 $\left.2^{\text {high }} / \mathrm{SUZ}_{2} 2^{\text {high }} / \mathrm{EED}^{\text {high }}\right)(26.4 \%$ vs $9.5 \%$, p = .009 ), and $\mathrm{H} 3 \mathrm{~K} 27 \mathrm{me} 3$ ( $40.6 \%$ vs $21.2 \%$; $\mathrm{p}=.004$ ). BCL2 was positively correlated with c-MYC ( $49.3 \%$ vs $26.8 \%$; $\mathrm{p}=.002$ ). These results are summarized in Fig. 2C. BCL2 expression showed no significant correlation with the tested PRC2-related markers.

The expression level of HOTAIR was significantly higher in cases of $\mathrm{EZH} 2^{\text {high }}$ than those of $\mathrm{EZH} 2^{\text {low }}$ (mean ratio value, 207 vs $66 ; \mathrm{p}=.027$ ) (Fig. 2D). For other markers, no statistical significance was observed according to HOTAIR expression level.

\section{HOTAIR expression in in relation to clinicopathological variables of DLBCL}

In the present study, 23.8\% of cases (39/164) showed high HOTAIR expression levels $\left(\right.$ HOTAIR $\left.^{\text {high }}\right)$. In the correlation analysis between HOTAIR ${ }^{\text {high }}$ and clinicopathological characteristics of DLBCL (Table 1), high expression of c-MYC protein was less frequent in HOTAIR ${ }^{\text {high }}$ than in $\operatorname{HOTAIR}^{\text {low }}(\mathrm{p}=.037)$. The rate of co-expression of c-MYC and BCL2 was significantly lower in HOTAIR ${ }^{\text {high }}$ than in $\operatorname{HOTAIR}^{\mathrm{low}}(\mathrm{p}=.015)$. Other clini- copathological factors including EBV ( $\mathrm{p}=.660)$ and Hans classification $(\mathrm{p}=.746)$ showed no significant correlation with HOTAIR expression (Table 1).

\section{Clinical significance of RNA and protein expression}

In univariate analysis for overall survival of DLBCL patients (Table 2), the known prognostic factors including age $>60$ ( $\mathrm{p}<$ .001), Eastern Cooperative Oncology Group performance status $\geq 2$ ( $\mathrm{p}<.001)$, elevated lactate dehydrogenase $(\mathrm{p}<.001)$, extranodal sites $\geq 2(\mathrm{p}=.001)$, Ann-Arbor stage III-IV ( $\mathrm{p}=.003)$, and International Prognostic Index (IPI) risk $\geq 3(\mathrm{p}<.001)$ showed significant association with reduced overall survival rate. Expression of c-MYC and co-expression of c-MYC/BCL2 were also related to inferior overall survival rate $(\mathrm{p}=.032$ and $\mathrm{p}=.006$, respectively). Among the PRC pathway markers (EZH2, SUZ12, EED, and H3K27me3), SUZ12 $2^{\text {high }}$ and $\mathrm{H} 3 \mathrm{~K} 27 \mathrm{me}^{\text {high }}$ were related to inferior overall survival rate $(\mathrm{p}=.028$ and $\mathrm{p}=.010$, respectively) (Fig. 3A, B). HOTAIR ${ }^{\text {high }}$, however, was significantly related to superior overall survival rate than $\operatorname{HOTAIR}^{\text {low }}(\mathrm{p}=$ .004) (Fig. 3C).

In multivariate analysis (Table 2), $\mathrm{H} 3 \mathrm{~K} 27 \mathrm{me}^{3^{\text {high }}}$ revealed an independent effect on poor overall survival (hazard ratio, 2.0; $\mathrm{p}=$ $.023)$. IPI risk $\geq 3$ was still determined as an independent prognostic factor (hazard ratio, 3.5; $\mathrm{p}<.001$ ), while HOTAIR ${ }^{\text {high }}$ showed a tendency to be related to improved survival with marginal significance (hazard ratio, $0.5 ; \mathrm{p}=.086$ ).

\section{DISCUSSION}

In our previous study, high level of global H3K27me3 was found to be a negative prognostic indicator in patients with DLBCL. ${ }^{8}$ This subsequent study aimed to explore the role of HOTAIR, possibly functioning via induction of $\mathrm{H} 3 \mathrm{~K} 27 \mathrm{me} 3$. We
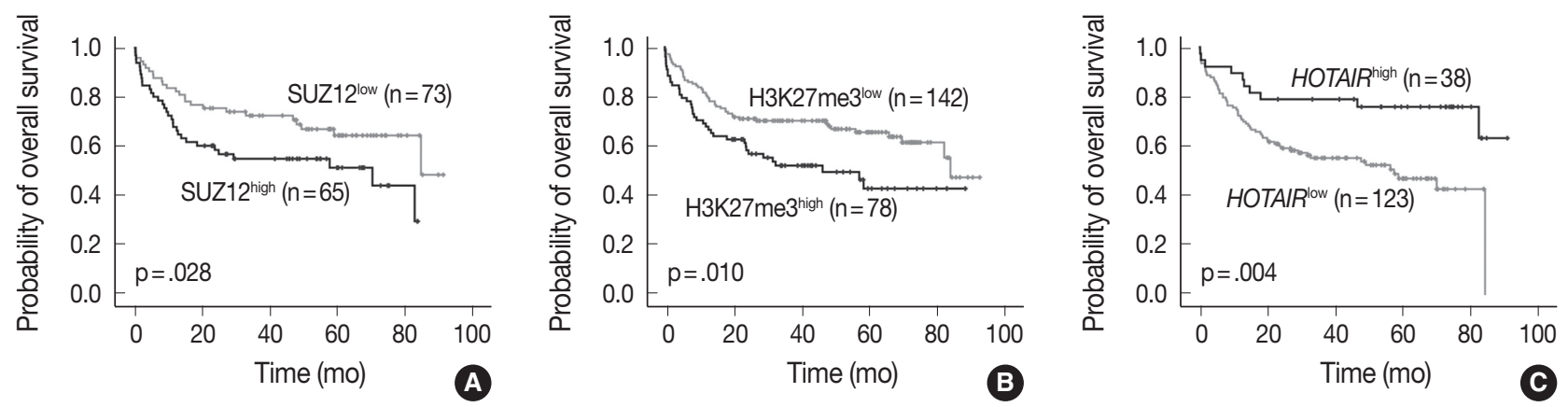

Fig. 3. Overall survival based on SUZ12 (A), H3K27me3 (B), and HOTAIR expression (C). High expression of SUZ12 (SUZ12 ${ }^{\text {high }}$ ) and H3K27me3 (H3K27me3 ${ }^{\text {high})}$ is related to inferior overall survival, while high expression of HOTAIR (HOTAIR ${ }^{\text {high}) ~ i s ~ c o r r e l a t e d ~ w i t h ~ l o n g e r ~ o v e r a l l ~ s u r-~}$ vival than low levels of HOTAIR expression (HOTAIR lom). SUZ12, suppressor of zeste 12 homolog; HOTAIR, hox transcript antisense intergenic RNA. 
Table 1. Clinicopathological characteristics, relation to outcome, and association with HOTAIR level in DLBCL patients

\begin{tabular}{|c|c|c|c|c|}
\hline \multirow{2}{*}{ Variable } & \multirow{2}{*}{ No. $(\%)(n=164)$} & \multicolumn{2}{|c|}{ HOTAIR } & \multirow{2}{*}{ p-value } \\
\hline & & HOTAIR low & HOTAIR high & \\
\hline \multicolumn{5}{|l|}{ Sex } \\
\hline Male & $98(59.8)$ & $73(58.4)$ & $25(64.1)$ & .526 \\
\hline Female & $66(40.2)$ & $52(41.6)$ & $14(35.9)$ & \\
\hline \multicolumn{5}{|l|}{ Age (yr) } \\
\hline$\leq 60$ & $94(57.3)$ & 69 (55.2) & $25(64.1)$ & .326 \\
\hline$>60$ & $70(42.7)$ & $56(44.8)$ & $14(35.9)$ & \\
\hline \multicolumn{5}{|c|}{ ECOG performance status ${ }^{a}$} \\
\hline$<2$ & $132(80.5)$ & $96(76.8)$ & 36 (92.3) & .033 \\
\hline$\geq 2$ & $32(19.5)$ & 29 (23.2) & $3(7.7)$ & \\
\hline \multicolumn{5}{|l|}{ Ann-Arbor stage ${ }^{a}$} \\
\hline $\mid-\|$ & 89 (54.6) & $63(50.8)$ & $26(66.7)$ & .083 \\
\hline III-IV & $74(45.4)$ & 61 (49.2) & $13(33.3)$ & \\
\hline \multicolumn{5}{|l|}{ Extranodal sites ${ }^{a}$} \\
\hline$<2$ & $108(66.3)$ & 81 (65.3) & 27 (69.2) & .653 \\
\hline$\geq 2$ & $55(33.7)$ & $43(34.7)$ & $12(30.8)$ & \\
\hline \multicolumn{5}{|l|}{ Lactate dehydrogenase ${ }^{a}$} \\
\hline Normal & $90(55.2)$ & $65(52.4)$ & $25(64.1)$ & .201 \\
\hline Elevated & $73(44.8)$ & $59(47.6)$ & 14 (35.9) & \\
\hline IPI risk group ${ }^{a}$ & & $79(63.7)$ & $28(71.8)$ & .354 \\
\hline $0-2$ & $107(65.6)$ & 45 (36.3) & 11 (28.2) & \\
\hline $3-5$ & $56(34.4)$ & & & \\
\hline \multicolumn{5}{|l|}{ Hans classification $^{a}$} \\
\hline GCB & $44(28.0)$ & $32(27.1)$ & $12(30.8)$ & .660 \\
\hline Non-GCB & $113(72.0)$ & 86 (72.9) & 27 (69.2) & \\
\hline \multicolumn{5}{|l|}{ EBV } \\
\hline Negative & $132(88.0)$ & 98 (87.5) & 34 (89.5) & .746 \\
\hline Positive & $18(12.0)$ & 14 (12.5) & $4(10.5)$ & \\
\hline \multicolumn{5}{|l|}{$\mathrm{BCL}^{\mathrm{a}}$} \\
\hline Negative expression & $84(58.7)$ & $58(54.7)$ & 26 (70.3) & .098 \\
\hline Positive expression & 59 (41.3) & 48 (45.3) & $11(29.7)$ & \\
\hline \multicolumn{5}{|l|}{ c-MYCa } \\
\hline Negative expression & $92(62.2)$ & $63(57.3)$ & $29(76.3)$ & .037 \\
\hline Positive expression & $56(32.8)$ & 47 (42.7) & $9(23.7)$ & \\
\hline \multicolumn{5}{|l|}{ c-MYC/BCL2 ${ }^{\mathrm{a}}$} \\
\hline No co-expression & $110(77.5)$ & 76 (72.4) & $34(91.9)$ & .015 \\
\hline Co-expression & $32(22.5)$ & $29(27.6)$ & $3(8.1)$ & \\
\hline
\end{tabular}

Values are presented as number (\%).

HOTAIR, hox transcript antisense intergenic RNA; DLBCL, diffuse large B-cell lymphoma; ECOG, Eastern Cooperative Oncology Group; IPI, International Prognostic Index; GCB, germinal center B-like; EBV, Epstein-Barr virus.

alnformation not available in some cases.

found the positive correlation between PRC2 proteins, global $\mathrm{H} 3 \mathrm{~K} 27$ me3 levels, and c-MYC in DLBCL as expected.

In the present study, the positive correlation among PRC2 proteins (EZH2, SUZ12, and EED), global H3K27me3 levels, and c-MYC was also confirmed in DLBCL as expected. In addition, HOTAIR expression was related to EZH2 expression. These findings could support that the lncRNA HOTAIR may be involved in inducing $\mathrm{H} 3 \mathrm{~K} 27 \mathrm{me} 3$ through recruiting polycomb repressive complex, the methyltransferase EZH2 and core accessory proteins, SUZ12 and EED.
Based on many other studies, interaction between c-MYC and PRC2 (EZH2, SUZ12, and EED) is well known in the tumorigenesis of various cancer types including lymphomas. Protein c-MYC interacts with EZH2 as well as SUZ12/EED, and they induce the histone modification of $\mathrm{H} 3 \mathrm{~K} 27 \mathrm{me} 3$ on the promoter of target genes, which then represses gene expression. Many other studies have shown that EZH2 and c-MYC activate each other. ${ }^{13-15}$ From the present findings, it could be also suggested that c-MYC may be the possible mechanism for inducing H3K27me3 via PRC2-related pathways in DLBCLs. 
Table 2. Cox proportional hazard analyses of overall survival

\begin{tabular}{|c|c|c|c|c|c|}
\hline Variable & Category & $\begin{array}{c}\text { Univariate analysis } \\
\text { p-value }\end{array}$ & $\begin{array}{c}\text { Multivariate analysis } \\
\text { p-value }\end{array}$ & $\mathrm{HR}$ & $95 \% \mathrm{Cl}$ \\
\hline Age & $>60$ vs $\leq 60$ & $<.001$ & - & - & - \\
\hline ECOG PS & $\geq 2$ vs $<2$ & $<.001$ & - & - & - \\
\hline Ann-Arbor stage & III-IV vs I-II & .003 & - & - & - \\
\hline Extranodal sites & $\geq 2$ vs $<2$ & .001 & - & - & - \\
\hline Lactate dehydrogenase & Elevated vs normal & $<.001$ & - & - & - \\
\hline IPI risk group & $3-5$ vs $0-2$ & $<.001$ & $<.001$ & 3.5 & $1.9-6.3$ \\
\hline Hans classification & Non-GCB vs GCB & .16 & - & - & - \\
\hline EBV & Positive vs negative & .611 & - & - & - \\
\hline BCL2 & Positive vs negative & .076 & - & - & - \\
\hline C-MYC & Positive vs negative & .032 & - & - & - \\
\hline C-MYC/BCL2 & Co-expression vs no co-expression & .006 & .528 & 1.2 & $0.6-2.4$ \\
\hline EZH2 & High vs non-high & .883 & - & - & - \\
\hline SUZ12 & High vs non-high & .028 & .183 & 1.5 & $0.8-2.7$ \\
\hline EED & High vs non-high & .25 & - & - & - \\
\hline H3K27me3 & High vs non-high & .01 & .023 & 2 & $1.1-3.6$ \\
\hline HOTAIR & High vs non-high & .004 & .086 & 0.5 & $0.2-1.1$ \\
\hline
\end{tabular}

HR, hazard ratio; Cl, confidence interval; ECOG PS, Eastern Cooperative Oncology Group performance status; IPI, International Prognostic Index; EBV, Epstein-Barr virus; EZH2, enhancer of zeste homolog 2; SUZ12, suppressor of zeste 12 homolog; EED, embryonic ectoderm development; HOTAIR, hox transcript antisense intergenic RNA.

In other studies of solid tumors originating from various organs, high expression of HOTAIR showed a close association with poor prognosis. ${ }^{5}$ In the present study, however, high levels of HOTAIR expression showed an association with good prognosis in DLBCL, although the impact was not significant in the multivariate analysis. There are no comparable reports to support the present findings because this is the first study for the expression status of HOTAIR in hematologic malignancies.

Although interaction between c-MYC and HOTAIR has not been established in hematologic malignancies, a close relationship between them may be plausible when considering the close association of PRC2 and HOTAIR, and PRC2 and c-MYC. However, expression of c-MYC showed a negative correlation with HOTAIR in the present study. One of the possible reason is autoregulation of c-MYC reported in one study; c-MYC represses itself via forming autoregulatory loops with $\mathrm{EZH}^{2,13}$ therefore, HOTAIR might be involved in that process via EZH2. Whether HOTAIR expression is directly linked to the suppression of c-MYC or $\mathrm{H} 3 \mathrm{~K} 27 \mathrm{me} 3$ should be investigated in further functional studies. In the HOTAIR-related suppression of cMYC, PRC2-associated histone modification (H3K27me3) might be induced in the promoter region of $c-M Y C$ gene. For confirmation of this possible modulation, further study should be followed.

The favorable outcome of DLBCLs with high HOTAIR expression might be associated with the negative correlation with cMYC and/or BCL2. Recent evidence has shown that co-expres- sion of C-MYC and BCL2 proteins is associated with poor prognosis in DLBCL patients regardless of gene signature. ${ }^{11,12}$ This was also observed in the present study in the univariate survival analysis. When the factor of HOTAIR expression was added in the multivariate analysis; however, the prognostic effect of coexpression of c-MYC and BCL2 became weak, and only the factor of high $\mathrm{H} 3 \mathrm{~K} 27 \mathrm{me} 3$ level was important as well as the IPI risk score. $\mathrm{H} 3 \mathrm{~K} 27 \mathrm{me} 3$, the chromatin modification status induced via changes of HOTAIR, c-MYC, or other various known and unknown mechanisms, seems to be the most important factor in determining the fate of disease aggressiveness of DLBCL. Though little is known about the key role of HOTAIR in the malignant lymphoma, HOTAIR might be involved in regulation of various genes in the lymphomagenesis. Further study should follow to determine the explicit mechanism among the sophisticated modulatory networks of c-MYC, PRC2, H3K27me3, and HOTAIR.

Recent evidence has shown that inhibition of EZH2 methyltransferase activity provides a potential target therapy for EZH2deregulated lymphomas. In addition, the pharmacological inhibition of EZH2 activity results in a decrease of global H3K27me3 levels and a reactivation of silenced $P R C 2$ gene targets, and it inhibits growth of DLBCL cells. ${ }^{16,17}$ From these overall findings as well as the present findings, HOTAIR may also be used in a target therapy by modulating the c-MYC-EZH2/PRC2 loop in a subset of DLBCLs with a high level of H3K27me3.

In conclusion, we found frequent expression of PRC2 pro- 
teins and $\mathrm{H} 3 \mathrm{~K} 27$ me3 and positive correlation between these proteins and c-MYC in DLBCLs. High expression of H3K27me3 was determined as an independent predictor of poor prognosis, however, HOTAIR was associated with favorable overall survival, which can be partly explained by negative correlation with c-MYC. LncRNA HOTAIR expression could be one of the possible mechanisms to be involved in aggressive behavior of DLB$\mathrm{CL}$ via induction of $\mathrm{H} 3 \mathrm{~K} 27 \mathrm{me} 3$ and EZH2-related PRC2 activation.

\section{Conflicts of Interest}

No potential conflict of interest relevant to this article was reported.

\section{Acknowledgments}

This work was supported by grant no. 2014-01 from the Korean Medical Women's Association.

\section{REFERENCES}

1. Spizzo R, Almeida MI, Colombatti A, Calin GA. Long non-coding RNAs and cancer: a new frontier of translational research? Oncogene 2012; 31: 4577-87.

2. Wahlestedt C. Targeting long non-coding RNA to therapeutically upregulate gene expression. Nat Rev Drug Discov 2013; 12: 433-46.

3. Gupta RA, Shah N, Wang KC, et al. Long non-coding RNA HOTAIR reprograms chromatin state to promote cancer metastasis. Nature 2010; 464: 1071-6.

4. Qiu MT, Hu JW, Yin R, Xu L. Long noncoding RNA: an emerging paradigm of cancer research. Tumour Biol 2013; 34: 613-20.

5. Zhang S, Chen S, Yang G, et al. Long noncoding RNA HOTAIR as an independent prognostic marker in cancer: a meta-analysis. PLoS One 2014; 9: e105538.

6. Martin-Perez D, Piris MA, Sanchez-Beato M. Polycomb proteins in hematologic malignancies. Blood 2010; 116: 5465-75.

7. Bracken AP, Helin K. Polycomb group proteins: navigators of lin- eage pathways led astray in cancer. Nat Rev Cancer 2009; 9: 773-84. 8. Oh EJ, Yang WI, Cheong JW, Choi SE, Yoon SO. Diffuse large B-cell lymphoma with histone $\mathrm{H} 3$ trimethylation at lysine 27: another poor prognostic phenotype independent of c-Myc/Bcl2 coexpression. Hum Pathol 2014; 45: 2043-50.

9. Swerdlow SH, Campo E, Harris NL, et al. WHO classification of tumours of haematopoietic and lymphoid tissues. 4th ed. Lyon: IARC Press, 2008.

10. Hans CP, Weisenburger DD, Greiner TC, et al. Confirmation of the molecular classification of diffuse large B-cell lymphoma by immunohistochemistry using a tissue microarray. Blood 2004; 103: 275-82.

11. Johnson NA, Slack GW, Savage KJ, et al. Concurrent expression of MYC and BCL2 in diffuse large B-cell lymphoma treated with rituximab plus cyclophosphamide, doxorubicin, vincristine, and prednisone. J Clin Oncol 2012; 30: 3452-9.

12. Hu S, Xu-Monette ZY, Tzankov A, et al. MYC/BCL2 protein coexpression contributes to the inferior survival of activated B-cell subtype of diffuse large B-cell lymphoma and demonstrates high-risk gene expression signatures: a report from The International DLBCL Rituximab-CHOP Consortium Program. Blood 2013; 121: 4021-31.

13. Benetatos L, Vartholomatos G, Hatzimichael E. Polycomb group proteins and MYC: the cancer connection. Cell Mol Life Sci 2014; 71: 257-69.

14. Sander S, Bullinger L, Klapproth K, et al. MYC stimulates EZH2 expression by repression of its negative regulator miR-26a. Blood 2008; 112: 4202-12.

15. Neri F, Zippo A, Krepelova A, Cherubini A, Rocchigiani M, Oliviero S. Myc regulates the transcription of the $P R C 2$ gene to control the expression of developmental genes in embryonic stem cells. Mol Cell Biol 2012; 32: 840-51.

16. McCabe MT, Ott HM, Ganji G, et al. EZH2 inhibition as a therapeutic strategy for lymphoma with EZH2-activating mutations. Nature 2012; 492: 108-12.

17. Qi W, Chan H, Teng L, et al. Selective inhibition of Ezh2 by a small molecule inhibitor blocks tumor cells proliferation. Proc Natl Acad Sci U S A 2012; 109: 21360-5. 